\title{
Momordica charantia (bitter melon) reduces plasma apolipoprotein B-100 and increases hepatic insulin receptor substrate and phosphoinositide-3 kinase interactions
}

\author{
Pratibha V. Nerurkar ${ }^{1}$, Yun Kyung Lee ${ }^{1}$, Megan Motosue ${ }^{1}$, Khosrow Adeli ${ }^{2}$ and Vivek R. Nerurkar ${ }^{3}$ \\ ${ }^{1}$ Laboratory of Metabolic Disorders and Alternative Medicine, Department of Molecular Biosciences and Bioengineering, \\ College of Tropical Agriculture and Human Resources, University of Hawaii at Manoa, Honolulu, HI 96816, USA \\ ${ }^{2}$ Research Institute, The Hospital for Sick Children, University of Toronto, Toronto, M5G 1 X8 Canada \\ ${ }^{3}$ Retrovirology Research Laboratory, Department of Tropical Medicine, Medical Microbiology and Pharmacology, Asia-Pacific \\ Institute of Tropical Medicine and Infectious Diseases, John A. Burns School of Medicine, University of Hawaii at Manoa, \\ Honolulu, HI 96813, USA
}

(Received 24 July 2007 - Revised 18 January 2008 - Accepted 21 January 2008 - First published online 5 March 2008)

\begin{abstract}
Aqueous extracts or juice from unripened fruit of Momordica charantia (bitter melon) has traditionally been used in the treatment of diabetes and its complications. Insulin resistance is characterized by significant down-regulation of hepatic insulin signalling as documented by attenuated phosphorylation of insulin receptor (IR), IR substrates 1 and 2, phosphoinositide-3 kinase, protein kinase B, and over-expression of phosphotyrosine phosphatase 1B. We recently demonstrated that bitter melon juice (BMJ) is a potent inhibitor of apoB secretion and TAG synthesis and secretion in human hepatoma cells, HepG2, that may be involved in plasma lipid- and VLDL-lowering effects observed in animal studies. The aim of this study was to evaluate the effects of BMJ on plasma apoB levels and hepatic insulin signalling cascade in mice fed high-fat diet (HFD). Female C57BL/6 mice (4-6 weeks old) were randomized into three groups receiving regular rodent chow, HFD and HFD + BMJ. The data indicate that BMJ not only improves glucose and insulin tolerance but also lowers plasma apoB-100 and apoB-48 in HFD-fed mice as well as modulates the phosphorylation status of IR and its downstream signalling molecules. Investigating the biochemical and molecular mechanisms involved in amelioration of diabetic dyslipidaemia by BMJ may lead to identification of new molecular targets for dietary/alternative therapies.
\end{abstract}

Bitter melon: ApoB: Insulin receptor: Phosphorylation: Insulin receptor substrate: Phosphoinositide-3 kinase: Diabetes: Hyperlipidaemia

For the past two decades, obesity and associated metabolic disorders such as type 2 diabetes and CVD have been escalating worldwide. Major risk factors for CVD include insulin resistance, hypertension and diabetic dyslipidaemia. Hypertriglyceridaemia and hepatic overproduction of apoB and VLDL are among the most prevalent complications of insulin resistance and are associated with high risk of developing CVD. Currently, diabetes and dyslipidaemia treatments require a combination of drugs. There is no single drug treatment available that will treat both these disorders. Furthermore, available treatments are prone to various side-effects such as secondary weight gain, drug-drug interactions and secondary failure. It is therefore critical to identify new therapies that may influence lipid and glucose metabolism, have minimal side-effects and similar or more potent efficacies than conventional therapies.

Momordica charantia, also known as bitter melon (BM), is traditionally used in the treatment of diabetes and its complications $^{(1)}$. Animal studies also indicate additional effects of $\mathrm{BM}$ in regulating weight gain and lipid metabolism ${ }^{(2-7)}$.
Aqueous extracts of BM fruit and seeds have been demonstrated to reduce VLDL levels in normal control rats, and normalize elevated VLDL levels in diabetic rats ${ }^{(8)}$. The most fundamental molecular defect in insulin-resistant states is the resistance to cellular actions of insulin, particularly insulin-stimulated glucose uptake, which leads to hyperinsulinaemia, enhanced VLDL secretion and hypertriacylglycerolaemia. Assembly and secretion of VLDL is a complex process involving the interaction of apoB with both core and surface lipids to form a lipoprotein particle. Regulation of VLDL secretion by insulin is dependent on apoB synthesis and degradation, microsomal TAG transfer protein activity, apoB phosphorylation and/or apoB mRNA editing ${ }^{(9,10)}$. Insulin-mediated inhibition of apoB synthesis and secretion is further dependent on phosphoinositide-3 kinase (PI3K)-associated activation of protein kinase $\mathrm{B}(\mathrm{PKB} / \mathrm{Akt})^{(11)}$, while degradation of apoB in the endoplasmic reticulum/golgi is dependent only on PI3K phosphorylation ${ }^{(12)}$. Insulin resistance and dyslipidaemia are characterized by significant down-regulation of hepatic insulin signalling as documented by attenuated phosphorylation of IR, IR substrates

Abbreviations: Akt, protein kinase B; BM, bitter melon; BMJ, BM juice; HFD, high-fat diet; IR, insulin receptor; IRS-1, IR substrate 1; IRS-2, IR substrate 2; PI3K, phosphoinositide-3 kinase; PKB, protein kinase B; pTyr, tyrosine phosphorylation status.

* Corresponding author: Dr Pratibha V. Nerurkar, fax +1 808956 3582, email pratibha@hawaii.edu 
1 and 2 (IRS- 1 and IRS-2), PI3K and/or PKB/Akt ${ }^{(13)}$. In fructose-fed, insulin-resistant hamster model, Taghibiglou et al. ${ }^{(14)}$ demonstrated a direct link between attenuated hepatic insulin signalling and synthesis and secretion of VLDL apoB.

Our recent in vitro studies demonstrated that BM juice (BMJ) significantly reduces apoB secretion under basal and lipid-rich conditions, in human hepatoma cells, HepG $2^{(15)}$. Therefore, the aim of the present study was to evaluate the in vivo effects of BMJ on plasma apoB levels and the hepatic insulin signalling cascade in mice fed a high-fat diet (HFD). Our previous data indicate that BMJ lowers plasma apoB100 and apoB-48 in HFD-fed mice and modulates phosphorylation status of IR and its downstream signalling molecules.

\section{Methods}

\section{Preparation of bitter melon juice}

The Chinese variety of young BM (raw and green) was obtained from a local farmers' market, washed and deseeded. BMJ was extracted as published previously ${ }^{(7,15)}$. In brief, BMJ was extracted using a household juicer and centrifuged at $4500 \mathrm{rpm}$ at $4^{\circ} \mathrm{C}$ for $30 \mathrm{~min}$. Supernatant BMJ was freezedried at $-45^{\circ} \mathrm{C}$ for $72 \mathrm{~h}$ and stored at $-80^{\circ} \mathrm{C}$ until used for feeding studies. Physical properties and mineral contents of Chinese BM were analysed at the Agriculture Diagnostic Service Center at the University of Hawaii at Manoa, and total carotenoids were analysed at the Analytical Laboratory of the Cancer Research Center of Hawaii (Table 1). Values represent means and standard deviations of five independent analyses of BM samples obtained from a local farmers' market throughout the year. The amounts of crude protein found in the extracts are similar to those reported previously ${ }^{(16)}$.

Table 1. Physical properties and mineral contents of Chinese bitter melon juice (BMJ)

(Mean values and standard deviations)

\begin{tabular}{|c|c|c|c|c|}
\hline \multirow[b]{2}{*}{ Analysis } & \multicolumn{2}{|c|}{ BMJ $(n 5)$} & \multicolumn{2}{|c|}{$\begin{array}{l}\text { Lyophilized } \\
\text { BMJ (n 5) }\end{array}$} \\
\hline & Mean & SD & Mean & SD \\
\hline DM (\%) & $3 \cdot 0$ & 0.08 & $75 \cdot 0$ & 0.5 \\
\hline Ash (\%) & $14 \cdot 8$ & $1 \cdot 2$ & $14 \cdot 8$ & 0.03 \\
\hline Crude protein (\%) & $24 \cdot 3$ & 0.8 & $24 \cdot 7$ & 0.4 \\
\hline Crude fat (\%) & 1.9 & 0.04 & $2 \cdot 1$ & 0.01 \\
\hline \multicolumn{5}{|l|}{ Minerals } \\
\hline Phosphorus (\%) & 1.05 & 0.01 & 0.91 & 0.05 \\
\hline Potassium (\%) & $7 \cdot 9$ & 0.2 & 6.92 & 0.06 \\
\hline Calcium (\%) & 0.2 & 0.02 & $0 \cdot 14$ & 0.0 \\
\hline Magnesium (\%) & 0.06 & 0.002 & 0.52 & 0.003 \\
\hline Sodium (\%) & 0.8 & 0.05 & 0.3 & 0.002 \\
\hline Boron (ppm) & $13 \cdot 0$ & 0.8 & $13 \cdot 0$ & 0.6 \\
\hline Copper (ppm) & $21 \cdot 0$ & 0.02 & $19 \cdot 0$ & $1 \cdot 2$ \\
\hline Iron (ppm) & 148.0 & $6 \cdot 3$ & $96 \cdot 0$ & $5 \cdot 7$ \\
\hline Manganese (ppm) & $32 \cdot 0$ & $1 \cdot 1$ & $32 \cdot 0$ & $1 \cdot 2$ \\
\hline Molybdenum (ppm) & $2 \cdot 0$ & 0.03 & 1.0 & 0.0 \\
\hline Zinc (ppm) & $65 \cdot 0$ & $1 \cdot 3$ & $43 \cdot 0$ & 1.4 \\
\hline Total carotenoids $(\mathrm{mg} / 100 \mathrm{~g})$ & ND & ND & $48 \cdot 0$ & 2.5 \\
\hline
\end{tabular}

ND, not detected.

\section{Animals and treatment}

Female C57BL/6 mice (4-6 weeks old) were purchased from Jackson Laboratories (Ann Harbor, MI, USA) and randomized in three groups of seven to eight animals each: (1) control ( $n$ 7), (2) HFD ( $n$ 7) and (3) HFD $+1.5 \%$ freeze-dried BMJ (w/w, $n$ 8). Control rodent chow contained $4.8 \mathrm{~kJ} \%$ fat $(17.03 \mathrm{~kJ} / \mathrm{g}$ (4.07 kcal/g); \#D12329; Research Diets, New Brunswick, NJ, USA), whereas HFD chow contained $58 \mathrm{~kJ} \%$ fat $(23.4 \mathrm{~kJ} / \mathrm{g} \quad(5.6 \mathrm{kcal} / \mathrm{g})$; \#D12331; Research Diets). Nutrient contents of the two diets are depicted in Table 2. Lyophilized BMJ powder was manually mixed with the diets daily to obtain a homogenous mixture and fed to the animals. These studies were approved by the Institutional Board of the University of Hawaii Institutional Animal Care and Use Committee. All procedures were conducted in accordance with guidelines established by the National Institutes of Health. Animals were housed individually and allowed to eat and drink ad libitum for 16 weeks. Food and water intake was measured daily, while body weights were measured on consecutive days. At the beginning of the study, tail vein blood was used to determine glucose levels with the OneTouch Ultra ${ }^{\mathrm{TM}}$ glucometer (Lifescan, USA). Two weeks before the end of the study, glucose and insulin tolerance tests were performed after an overnight $12 \mathrm{~h}$ fast according to published protocols ${ }^{(17)}$. In brief, tolerance tests were performed by an intraperitoneal injection of either glucose $(1.5 \mathrm{mg} / \mathrm{g}$ body weight $)$ or insulin $(0.5 \mathrm{U} / \mathrm{kg}$ body weight). Tail blood was used to determine glucose levels at 0, 30, 60 and $120 \mathrm{~min}$ with the OneTouch Ultra ${ }^{\mathrm{TM}}$ glucometer. At the end of the study, after an overnight fast, mice in each group were anaesthetized by isofluorane, followed by blood draw by cardiac puncture for biochemical assays. For plasma preparation, whole blood was collected in heparinized tubes. Plasma TAG and cholesterol levels were measured using the Infinity TG Liquid Stable Reagent and Infinity Cholesterol Liquid Stable Reagent commercial kits (Thermo-DMA, St Louisville, CO, USA), according to the manufacturer's instructions, and absorbance was read at $540 \mathrm{~nm}$ using a Perkin-Elmer multiplate reader (Wallac Victor2; Perkin-Elmer Life Sciences). Plasma NEFA were determined using a commercial kit according to the manufacturer's protocol (Wako Chemicals Inc., Richmond, VA, USA). Liver function tests were analysed by measuring serum alanine amino transferase, aspartate aminotransaminase (Biotron Diagnostics USA Inc., Hemet, CA, USA) and lactate dehydrogenase (Bioassays Systems, Hayward, CA, USA). All tissues were snap-frozen in liquid nitrogen and stored at $-80^{\circ} \mathrm{C}$ until analysis.

\section{Analysis of hepatic TAG}

Hepatic TAG mass was analysed by the method of Scribner et al. ${ }^{(18)}$, with slight modifications. In brief, a $5 \%$ liver homogenate was prepared in sucrose buffer containing $0.3 \mathrm{~mm}$-sucrose, $25 \mathrm{nM}$-2-mercaptoethanol and $10 \mathrm{~mm}$-EDTA, $\mathrm{pH} 7 \cdot 0$, and mixed with hexane-isopropanol $(3: 2, \mathrm{v} / \mathrm{v})$. The organic phase was dried, resuspended in $100 \mu \mathrm{l}$ ethanol and TAG mass was enzymatically determined as mentioned previously $^{(15)}$. TAG mass was normalized to liver weights (mg TAG/g tissue). 
Table 2. Nutrient composition of control and high-fat diets*

\begin{tabular}{|c|c|c|c|c|c|c|c|c|c|c|}
\hline \multirow[b]{2}{*}{ Contents } & \multicolumn{5}{|c|}{ Control diet (\#D12329) } & \multicolumn{5}{|c|}{ High-fat diet (\#D12331) } \\
\hline & $g \%$ & kJ\% & $g$ & $\mathrm{~kJ}$ & kcal & $g \%$ & $\mathrm{~kJ} \%$ & $g$ & $\mathrm{~kJ}$ & kcal \\
\hline Carbohydrate & $74 \cdot 3$ & $73 \cdot 1$ & & & & 35.5 & $25 \cdot 5$ & & & \\
\hline Fat & $4 \cdot 8$ & $10 \cdot 5$ & & & & $35 \cdot 8$ & $58 \cdot 0$ & & & \\
\hline Total & & $100 \cdot 0$ & & & & & $100 \cdot 0$ & & & \\
\hline $\mathrm{kJ} / \mathrm{g}$ & $17 \cdot 03$ & & & & & $23 \cdot 26$ & & & & \\
\hline $\mathrm{kcal} / \mathrm{g}$ & 4.07 & & & & & $5 \cdot 56$ & & & & \\
\hline \multicolumn{11}{|l|}{ Ingredients } \\
\hline Casein, 80 Mesh & & & 228 & 3816 & 912 & & & 228 & 3816 & 912 \\
\hline DL-Methionine & & & 2 & 0 & 0 & & & 2 & 0 & 0 \\
\hline Maltodextrin 10 & & & 170 & 2845 & 680 & & & 170 & 2845 & 680 \\
\hline Maize starch & & & 0 & 0 & 0 & & & 0 & 0 & 0 \\
\hline Sucrose & & & 835 & 13975 & 3340 & & & 175 & 2929 & 700 \\
\hline Soyabean oil & & & 25 & 941 & 225 & & & 25 & 941 & 225 \\
\hline Coconut oil, hydrogenated & & & 40 & 1506 & 360 & & & 333.5 & $12558 \cdot 2$ & $3001 \cdot 5$ \\
\hline Sodium bicarbonate & & & $10 \cdot 5$ & 0 & 0 & & & 10.5 & 0 & 0 \\
\hline Potassium citrate, $1 \mathrm{H}_{2} \mathrm{O}$ & & & 4 & 0 & 0 & & & 4 & 0 & 0 \\
\hline Vitamin mix V10001 & & & 10 & 167 & 40 & & & 10 & 167 & 40 \\
\hline Choline bitartrate & & & 2 & 0 & 0 & & & 2 & 0 & 0 \\
\hline FD\&C Blue Dye no. 1 & & & 0.1 & 0 & 0 & & & 0 & 0 & 0 \\
\hline FD\&C Blue Dye no. 40 & & & 0 & 0 & 0 & & & $0 \cdot 1$ & 0 & 0 \\
\hline Total & & & $1366 \cdot 6$ & $23250 \cdot 0$ & $5557 \cdot 0$ & & & $1000 \cdot 1$ & $23256 \cdot 2$ & $5558 \cdot 5$ \\
\hline
\end{tabular}

*Research Diets (New Brunswick, NJ, USA).

\section{Analysis of plasma apolipoproteins}

Plasma apoB-100 and apoB-48 levels were determined using Western blotting techniques ${ }^{(19)}$. In brief, $15 \mu \mathrm{g}$ plasma proteins were separated on $7.5 \%$ polyacrylamide gels (Bio-Rad Laboratories, Hercules, CA, USA), transferred to nitrocellulose membrane at $80 \mathrm{~V}$ for $90 \mathrm{~min}$, washed and blocked in $1 \%$ BSA. Membranes were incubated overnight with goat polyclonal anti-mouse apoB (Santa Cruz Biotechnology Inc., Santa Cruz, CA, USA) and rabbit anti-mouse apoB 48/100 (BioDesign International, Saco, ME, USA) primary antibodies and with corresponding horseradish peroxidase-conjugated secondary antibodies for $2 \mathrm{~h}$. apoB-100 and apoB-48 were detected using a commercially available electrochemiluminescence kit (Amersham Biosciences, Piscataway, NJ, USA). Protein bands were scanned using the Molecular Imager Gel Doc XR System (Bio-Rad) and intensities were analysed using the Discovery Series ${ }^{\mathrm{TM}}$ Quantity One 1-D Analysis Software (Bio-Rad).

\section{Tyrosine phosphorylation status of insulin signalling proteins}

Tyrosine phosphorylation status (pTyr) of IR $\beta$, IRS-1 and IRS-2, and serine/threonine phosphorylation of Akt were analysed according to published protocols ${ }^{(9)}$. Liver homogenate $(10 \%)$ was prepared on ice in a specialized buffer $(150 \mathrm{mM}-$ $\mathrm{NaCl}, 10 \mathrm{~mm}$-tris(hydroxymethyl)aminomethane, $\mathrm{pH}$ 7.4, 1 mM-EDTA, 1 mM-ethyleneglycol-bis-(aminoethylether) $N$, $N, N^{\prime}, N^{\prime}$-tetraacetic acid, $1 \%$ Triton $\mathrm{X}-100,1 \%$ Nonidet $\mathrm{P}$ 40, 2 mM-phenylmethylsulphonyl fluoride, $100 \mathrm{~mm}$-sodium fluoride, $10 \mathrm{~mm}$-sodium pyrophosphate and $2 \mathrm{mM}$-sodium orthovanadate and protease inhibitors (Complete ${ }^{\mathrm{TM}}$; Boehringer Mannheim Corp., Indianapolis, IN, USA)). Protein concentrations were determined using Bradford protein assay reagent according to the manufacturer's instructions
(Bio-Rad Laboratories) and $0.5 \mathrm{mg}$ proteins was subjected to overnight immunoprecipitation with specific polyclonal antibodies against either IR $\beta$-subunit, IRS-1, IRS- 2 or Akt $(1 \mu \mathrm{g}$ antibody $/ 0.5 \mathrm{mg}$ total cell lysate) and $10 \%$ protein A-Sepharose. Immunoprecipitates were then washed with ice-cold PBS containing $100 \mathrm{~mm}$-sodium fluoride, $10 \mathrm{~mm}$-sodium pyrophosphate, 2 mM-sodium orthovanadate, $0 \cdot 1 \%$ Nonidet P 40 and $0.1 \%$ Triton $\mathrm{X}-100$.

Immunoprecipitates were separated on $7.5 \%$ polyacrylamide gels (Bio-Rad) and were electrophoretically transferred on to nitrocellulose membranes. The membranes were blocked with $5 \%$ milk, incubated with either monoclonal antibody apY (1:1000 dilution) or phosphor-serine/threonine antibody (Santa Cruz Biotechnology), washed and probed with a secondary antibody conjugated to horseradish peroxidase (Santa Cruz Biotechnology). Proteins were visualized with an enhanced electrochemiluminescence detection kit and band intensities were analysed as described earlier. The intensity of IR- $\beta$, IRS- 1 and IRS- 2 bands was initially normalized against $\beta$-actin and the normalized values of the protein of interest were used to adjust the ratio of the experimental phosphorylated bands.

\section{Analysis of PPAR $\alpha$ proteins}

Nuclear fractions were isolated from frozen livers using the NXTRACT, CellLytic NuCLEAR Extraction Kit (Sigma, St. Louis, MO, USA) according to the manufacturer's protocol. Nuclear proteins $(30 \mu \mathrm{g})$ were loaded on a $10 \%$ polyacrylamide gel, transferred to nitrocellulose membrane, blocked in $10 \%$ milk for $2 \mathrm{~h}$, probed with anti-human PPAR $\alpha$ primary antibody overnight at room temperature (Affinity Bioreagent, Golden, CO, USA), and followed with anti-mouse IgG2b conjugated to horseradish peroxidase, for $2 \mathrm{~h}$ (Santa Cruz 
Biotechnology). Proteins were visualized by an electrochemiluminescence kit and band intensities were analysed as described earlier.

\section{Statistical analysis}

Statistical analysis was conducted using SAS software (SAS Institute, Cary, NC, USA). A one-way ANOVA model was used to compare means between the three animal groups (control, HFD and HFD + BMJ). For repeated assays of plasma concentrations of various blood analytes, a repeated measure ANOVA was used to compare means adjusted for the time interval at which samples were obtained. For each sample, duplicate determinations were performed. Appropriate transformation of the data was performed to normalize distributions and stabilize variances. Post hoc pair-wise multiple comparisons were evaluated using the Ryan-Einot-Gabriel-Welsch multiple range test. All $P$ values were based on two-sample tests. Results were considered significant at $P<0.05$.

\section{Results}

Effects of bitter melon juice on body weight and plasma chemistry

BMJ is not a part of the traditional rodent chow and has an extremely bitter taste. It is well-known that rodents are sometimes very sensitive to inclusion of 'unusual' taste in their diet that can reduce food intake. As observed in Table 3, supplementation with BMJ had no significant effect on the overall daily food intake, measured as $\mathrm{kJ} / \mathrm{d}$. HFD-fed mice started gaining weight after 4 weeks and as expected gained $110 \%$ more weight than control mice receiving regular chow $(P<0 \cdot 05)$. Although supplementation with BMJ resulted in $21 \%$ lower weight gain than HFD-fed mice, the body weights were still significantly higher than the control group (Table $3, P<0.05$ ). As previously reported, HFD significantly increased plasma glucose,
TAG, total cholesterol and hepatic TAG mass, while BMJ normalized the plasma glucose and lipids (Table 3) ${ }^{(20)}$. Similarly, BMJ normalized liver weight and lowered hepatic TAG levels by $30 \%$ in mice-fed HFD (Table 3, $P<0 \cdot 05)$. BMJ was observed to significantly lower increased serum aspartate aminotransaminase, alanine amino transferase and lactate dehydrogenase in HFD-fed mice (Table 3, $P<0.05$ ) and normalize the glucose (Fig. 1(A) and insulin tolerance tests (Fig. 1(B).

\section{Effects of bitter melon juice on plasma apoB}

To confirm our in vitro observations ${ }^{(15)}$, plasma apoB-100 and apoB-48 were analysed by Western blotting in mice fed control rodent chow (control), HFD and HFD + BMJ. As observed in Fig. 2, HFD significantly increased plasma apoB-100 levels by $103 \%$ and apoB-48 by $52 \%(P<0 \cdot 05)$, while BMJ normalized the plasma levels of both apolipoproteins.

\section{Effects of bitter melon juice on tyrosine phosphorylation of insulin receptor $\beta$ and insulin receptor substrate 1 and 2 proteins}

Neither HFD nor BMJ treatment had any significant effects on the abundance of hepatic IR $\beta$ (Fig. 3). Feeding of $58 \%$ fat diet for 16 weeks significantly reduced $\mathrm{p}$-Tyr of IR $\beta$ by $42 \%$ as compared to control $(P<0 \cdot 05)$, while BMJ significantly increased the p-Tyr levels of IR $\beta$ by $56 \%$ above that of control and $270 \%$ above that of HFD-fed mice (Fig. 3, $P<0.05$ ). Similarly, HFD significantly reduced p-Tyr IRS- 1 by $45 \%$ as compared to control, while BMJ significantly increased it by $84 \%$ above control and $334 \%$ above HFD-fed mice (Fig. 4(A), $P<0.05$ ). In contrast, HFD had no effect on IRS-2 phosphorylation, while BMJtreated mice demonstrated a significant increase by $55 \%$ above that of control (Fig. 5(A), $P<0.05$ ). Both, IRS-1 and IRS-2 mass were unaffected by HFD or HFD + BMJ (Figs. 4 and 5).

Table 3. Metabolic parameters in C57BL/6 female mice fed three different diets* (Mean values and standard deviations)

\begin{tabular}{|c|c|c|c|c|c|c|}
\hline \multirow[b]{2}{*}{ Variables } & \multicolumn{2}{|c|}{ Control ( $n 7)$} & \multicolumn{2}{|c|}{$\mathrm{HFD}(n 7)$} & \multicolumn{2}{|c|}{$\mathrm{HFD}+\mathrm{BMJ}(n 8)$} \\
\hline & Mean & SD & Mean & SD & Mean & SD \\
\hline Food intake $(\mathrm{kJ} / \mathrm{d})$ & $72 \cdot 0^{a}$ & $3 \cdot 8$ & $70 \cdot 7^{a}$ & $5 \cdot 0$ & $66 \cdot 1^{a}$ & $6 \cdot 3$ \\
\hline Food intake (kcal/d) & $17 \cdot 2^{\mathrm{a}}$ & 0.9 & $16 \cdot 9^{a}$ & $1 \cdot 2$ & $15 \cdot 8^{\mathrm{a}}$ & 1.5 \\
\hline Final body weight (g) & $19 \cdot 9^{a}$ & 0.9 & $41 \cdot 8^{b}$ & 1.9 & $33 \cdot 3^{c}$ & $1 \cdot 3$ \\
\hline Plasma glucose $(\mathrm{mg} / \mathrm{l})$ & $1121^{a}$ & 76 & $1666^{\mathrm{b}}$ & 171 & $1221^{a}$ & 157 \\
\hline Plasma TAG (mg/l) & $390^{a}$ & 116 & $1711^{\mathrm{b}}$ & 411 & $585^{a}$ & 140 \\
\hline Plasma cholesterol (mg/l) & $851^{a}$ & 89 & $1774^{b}$ & 261 & $1180^{\mathrm{a}}$ & 182 \\
\hline Plasma NEFA (mEq/l) & $0.4^{\mathrm{a}}$ & 0.02 & $0.7^{\mathrm{b}}$ & 0.04 & $0 \cdot 6^{\mathrm{a}}$ & 0.03 \\
\hline Liver weight $(\mathrm{g})$ & $1 \cdot 0$ & 0.1 & $1 \cdot 2^{b}$ & 0.08 & $1 \cdot 1^{\mathrm{b}}$ & 0.19 \\
\hline Liver TAG (mg/g tissue) & $110 \cdot 5^{a}$ & $2 \cdot 4$ & $180 \cdot 5^{b}$ & 3.7 & $123 \cdot 1^{\mathrm{a}}$ & $3 \cdot 2$ \\
\hline Serum AST (U/I) & $8 \cdot 2^{a}$ & 0.25 & $75 \cdot 3^{\mathrm{b}}$ & $5 \cdot 7$ & $25 \cdot 4^{\mathrm{a}}$ & 5.9 \\
\hline Serum ALT (U/I) & $10 \cdot 3^{a}$ & 1.4 & $48 \cdot 2^{b}$ & $9 \cdot 6$ & $18 \cdot 2^{\mathrm{a}}$ & $3 \cdot 3$ \\
\hline Serum LDH (U/I) & $95 \cdot 1^{a}$ & $15 \cdot 2$ & $273 \cdot 2^{b}$ & $45 \cdot 8$ & $120 \cdot 7^{a}$ & $17 \cdot 0$ \\
\hline
\end{tabular}

ALT, alanine amino transferase; AST, aspartate aminotransaminase; BMJ, bitter melon juice; HFD, high-fat diet; LDH, lactate dehydrogenase.

a,b,c Mean values within a row with unlike superscript letters were significantly different $(P<0.05)$.

* For details of procedures and diets, see Methods and Tables 1 and 2. 

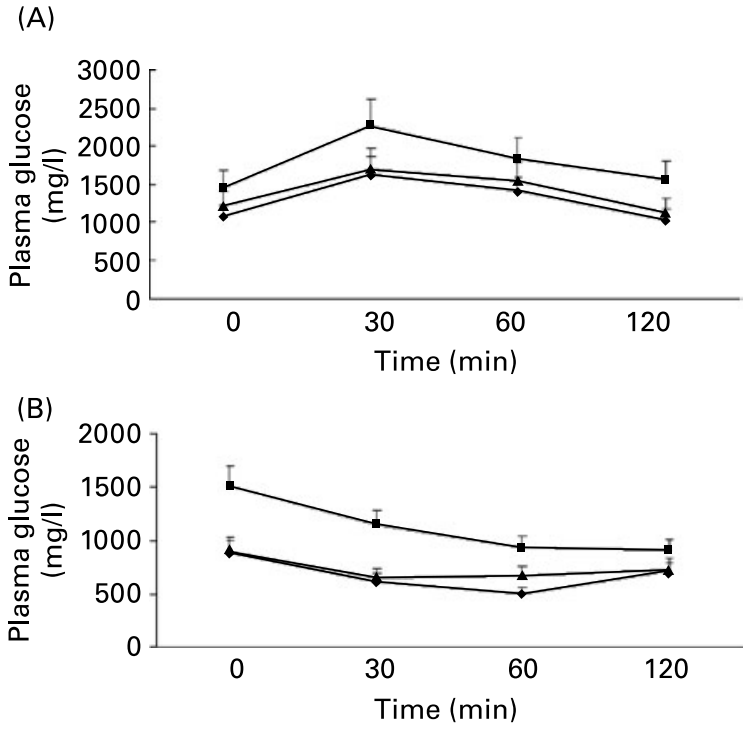

Fig. 1. Effects of bitter melon juice (BMJ) on plasma glucose tolerance $(A)$ and insulin tolerance $(B)$ in mice fed control rodent chow $(\diamond)$, high-fat diet $(\mathrm{HFD} ; \boldsymbol{\square})$ and HFD + BMJ $(\boldsymbol{\Lambda})$. Values are means with standard deviations depicted by vertical bars $(n 6)$. Mean values were significantly different from those of the control and HFD + BMJ group: ${ }^{\star} P<0.05$.

Effects of bitter melon juice on interactions between insulin receptor substrate and phosphoinositide-3 kinase

To evaluate the interactions between IRS-1/IRS-2 and PI3K, the IRS immunoprecipitates were separated on $7.5 \%$ SDS gels and the transferred proteins were probed with antibody against p85 and p110 subunits of PI3K. HFD significantly reduced the interactions between IRS-1 and p110 subunits of PI3K by $56 \%$, while BMJ increased it by $280 \%$ above HFD and $23 \%$ above control animals (Fig. 4 (B), $P<0.05$ ). In contrast, HFD did not affect the IRS-2 and p110 interaction, while BMJ increased it by $60 \%$ above both groups (Fig. 5(B),

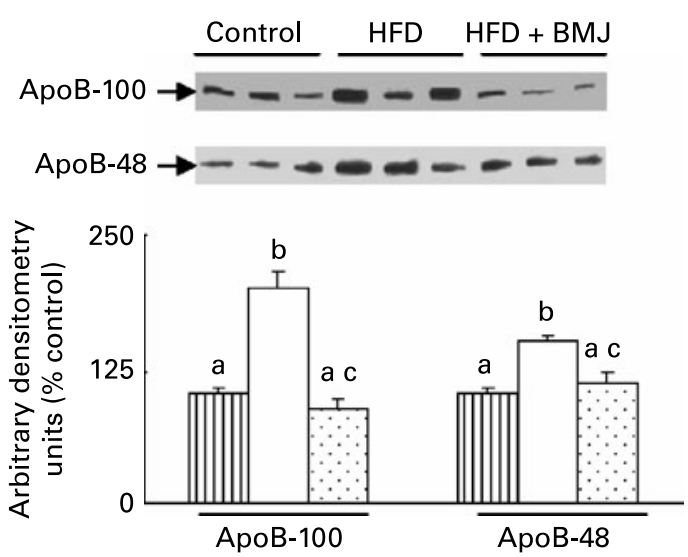

Fig. 2. Plasma apoB-100 and apoB-48 protein levels in mice fed high-fat diet (HFD, $\square$ ) and HFD + bitter melon juice (BMJ, $\square$; control, 四). The graph represents the densitometry scans of $220 \mathrm{kDa}$ (apoB-48) and $514 \mathrm{kDa}$ (apoB100 ) band intensities. Data are expressed as a percentage of control values, set at $100 \%$. Values are means with standard deviations depicted by vertical bars $(n 6){ }^{a, b, c}$ Mean values with unlike letters were significantly different $(P<0.05)$.
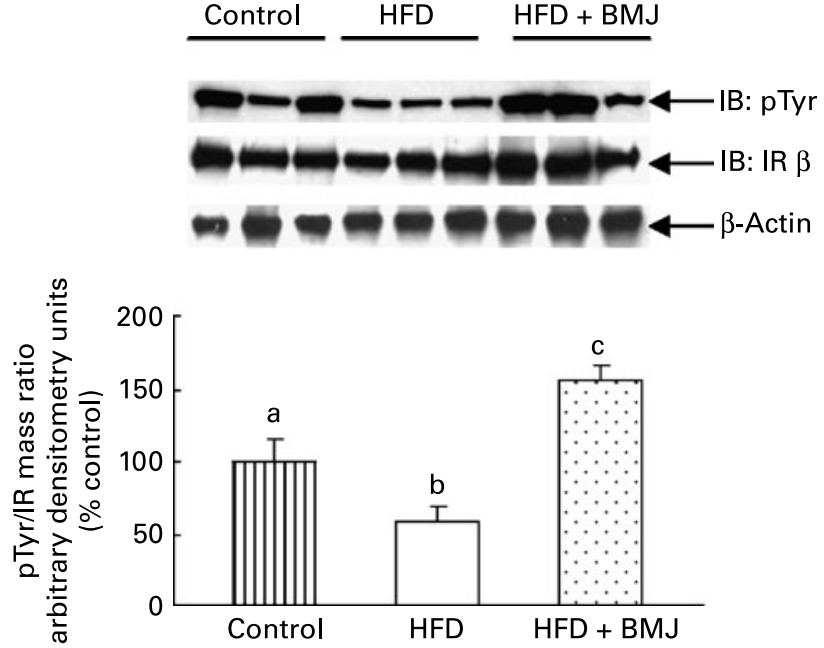

Fig. 3. Tyrosine phosphorylation (pTyr) of hepatic insulin receptor $\beta$ (IR $\beta$ ) in mice fed high-fat diet (HFD) and HFD + bitter melon juice (BMJ). The graph represents the densitometry scans of $95 \mathrm{kDa}$ band intensities. The $\beta$-actin band demonstrates equal amounts of protein loaded on the gel. Data are expressed as a percentage of control values, set at $100 \%$. Values are means with standard deviations depicted by vertical bars $(n 6) .{ }^{a, b, c}$ Mean values with unlike letters were significantly different $(P<0.05)$. IB, immunoblotting.

$P<0 \cdot 05)$. Neither HFD nor BMJ demonstrated any significant change in interactions between IRS-1/2 and p85 proteins as compared to controls (Figs. 4(C) and $5(\mathrm{C})$.

Effects of bitter melon juice on protein kinase B expression and serine 473/threonine 308 phosphorylation

Both, HFD and BMJ had no effect on either the Akt1 mass or serine 473/threonine 308 phosphorylation as compared to mice fed a control diet (data not shown). Similarly, HFD and BMJ did not affect Akt2 expression and its phosphorylation (data not shown).

\section{Effects of bitter melon juice on hepatic PPAR $\alpha$ protein expression}

Hepatic PAPR $\alpha$ proteins were analysed by Western blotting to test the hypothesis that BMJ normalizes the increased NEFA flux to the liver due to increased PAPR $\alpha$ expression in HFD-fed mice. As observed in Fig. 6, HFD significantly increased PPAR $\alpha$ protein expression by $156 \%$ above that of control, while feeding of BMJ significantly reduced it to control levels $(P<0.05)$.

\section{Discussion}

Increased VLDL production and apoB secretion in insulinresistant states confer a high risk of CVD and is likely a result of reduced sensitivity to insulin ${ }^{(21)}$. Since BM improves whole-body insulin sensitivity in animal studies ${ }^{(7,22)}$ and reduces apoB secretion in HepG2 cells ${ }^{(23)}$, we tested the hypothesis that $\mathrm{BMJ}$-associated apoB regulation in vivo was associated with modulation of the hepatic insulin signalling pathway. We present novel data indicating that BMJ can normalize plasma apoB-100 and apoB-48 in mice fed 
(A)
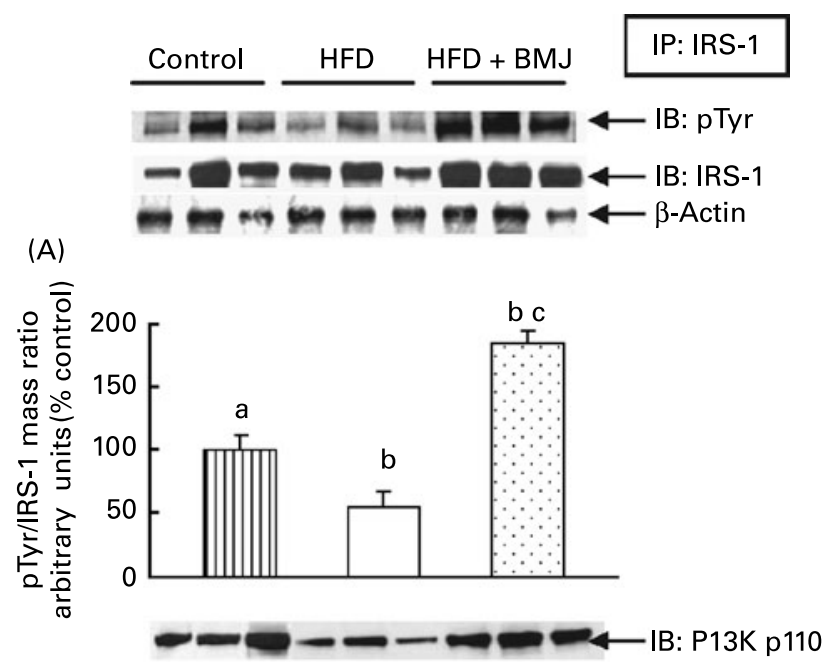

(B)
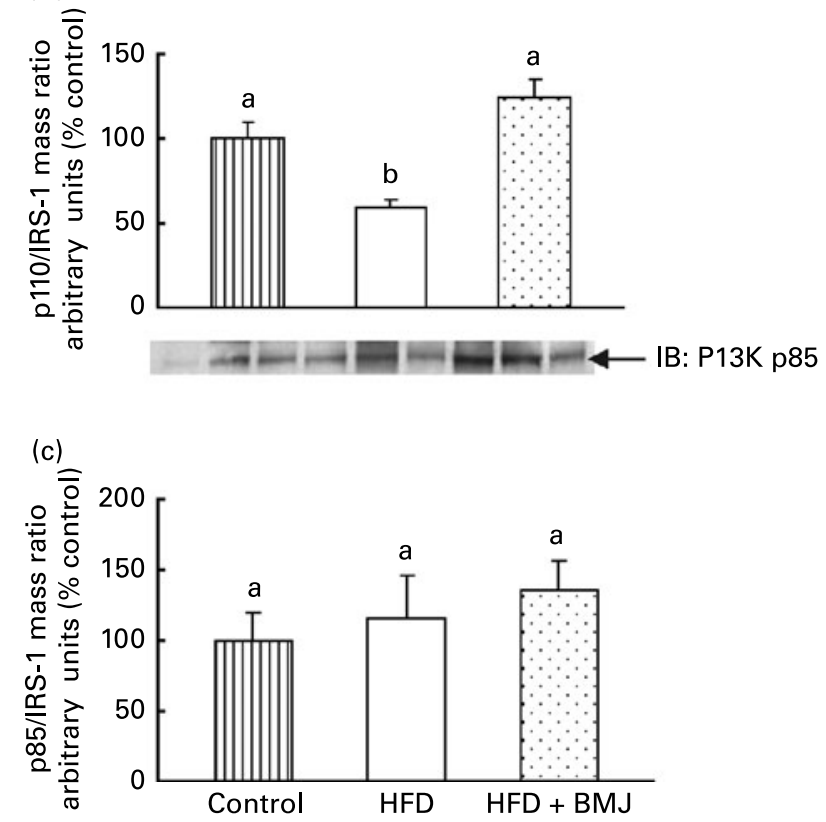

Fig. 4. Tyrosine phosphorylation (pTyr) of insulin receptor substrate 1 (IRS-1) and its interactions with p85 and p110 subunits of phosphoinositide-3 kinase (PI3K), in mice fed high-fat diet (HFD) and HFD + bitter melon juice (BMJ). The graph represents the densitometry scans of $180 \mathrm{kDa}$ (pTyr, IRS-1; A), $110 \mathrm{kDa}$ (p110/PI3K; B) and $85 \mathrm{kDa}$ (p85/PI3K; C) band intensity. The $\beta$-actin band demonstrates the amount of protein loaded on the gel. Data are expressed as a percentage of control values, set at $100 \%$. Values are means with standard deviations depicted by vertical bars $(n 6){ }^{a, b, c}$ Mean values with unlike letters were significantly different $(P<0.05)$. IP, immunoprecipitation; IB, immunoblotting.

a HFD for 16 weeks, possibly through modulation of the insulin signalling pathway.

Insulin is known to suppress apoB secretion via increasing PI3K activity and its association with IRS proteins. PI3K consists of a regulatory subunit, p85, and catalytic domain, p110, and plays a critical role not only in glucose, but also in apoB metabolism $^{(24)}$. In HFD-fed mice, hepatic insulin resistance was evident by reduced phosphorylation of hepatic IR $\beta$ and IRS-1, as well as the decreased association between IRS-1 and the catalytic domain of PI3K, p110, and elevated plasma glucose. BMJ significantly reduced plasma apoB-100
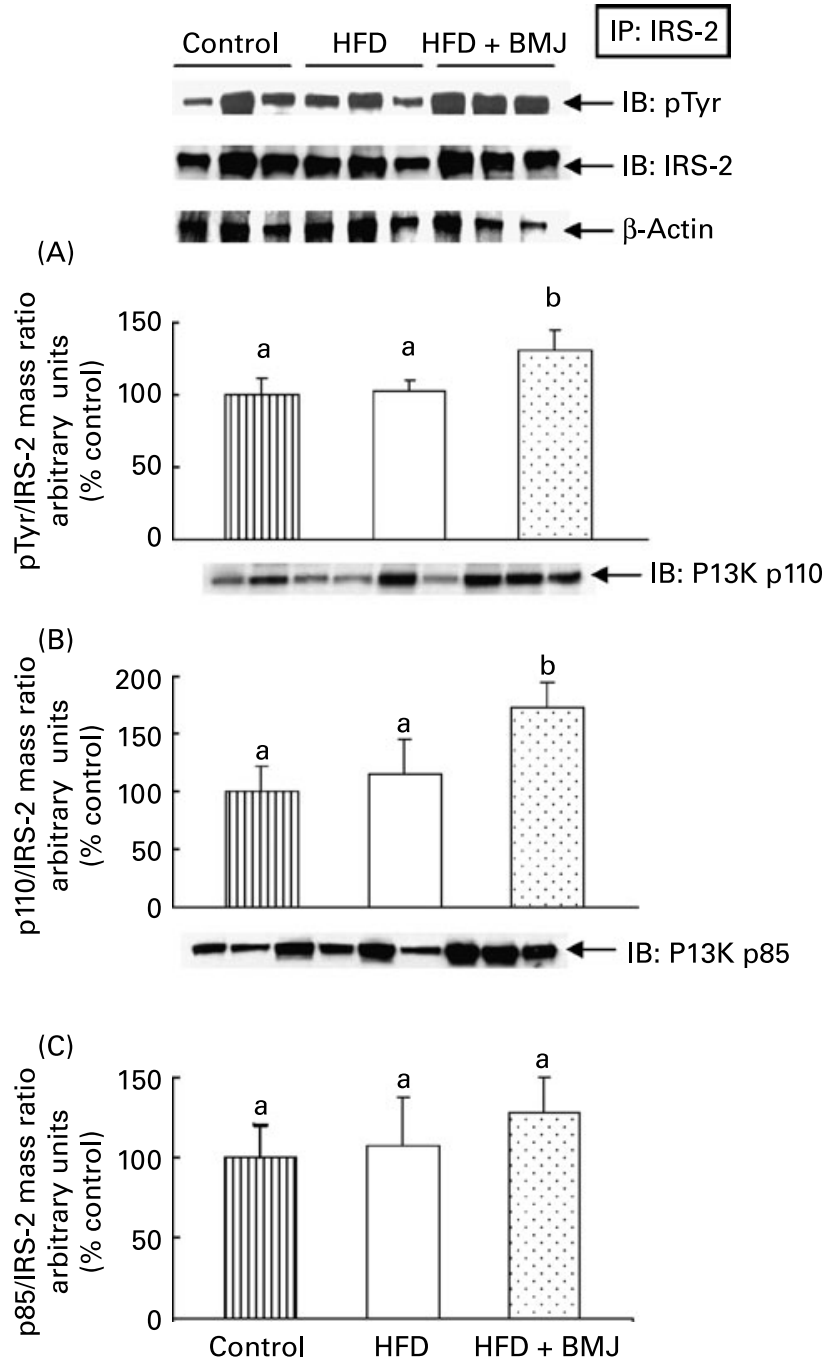

Fig. 5. Tyrosine phosphorylation (pTyr) of insulin receptor substrate 2 (IRS-2) and its interactions with $\mathrm{p} 85$ and $\mathrm{p} 110$ subunits of phosphoinositide-3 kinase (PI3K), in mice fed high-fat diet (HFD) and HFD + bitter melon juice $(B M J)$. The graph represents the densitometry scans of $180 \mathrm{kDa}$ (pTyr, IRS-2; A), $110 \mathrm{kDa}$ (p110/PI3K; B) and $85 \mathrm{kDa}$ (p85/PI3K; C) band intensities. The $\beta$-actin band demonstrates the amount of protein loaded on the gel. Data are expressed as a percentage of control values, set at $100 \%$. Values are means with standard deviations depicted by vertical bars $(n 6) .{ }^{a, b}$ Mean values with unlike letters were significantly different $(P<0.05)$. IP, immunoprecipitation; IB, immunoblotting.

and apoB-48 in HFD-fed mice treated with BMJ. In our previous in vitro study, BMJ-induced reduction in apoB secretion may have been caused by a lack of lipid bioavailability and/or impaired lipidation process during lipoprotein assembly ${ }^{(15)}$. In the current study, feeding of BMJ increased tyrosine phosphorylation of IR $\beta$ and activated the downstream signalling cascade as evident by increased tyrosine phosphorylation of IRS-1 and IRS-2 and their increased association with the catalytic subunit of PI3K, p110, due to its insulin-mimetic $\operatorname{activity}^{(1)}$. Under normal physiological conditions, p85 exists in stoichiometric excess of p110 subunit. However, mice lacking various isoforms of the $\mathrm{p} 85 \alpha$ or $\mathrm{p} 85 \gamma$ subunit of PI3K paradoxically demonstrate increased insulin sensitivity, due to improved PI3K signalling downstream of IRS proteins ${ }^{(25)}$. Studies suggest that the free p85 monomers bind to 

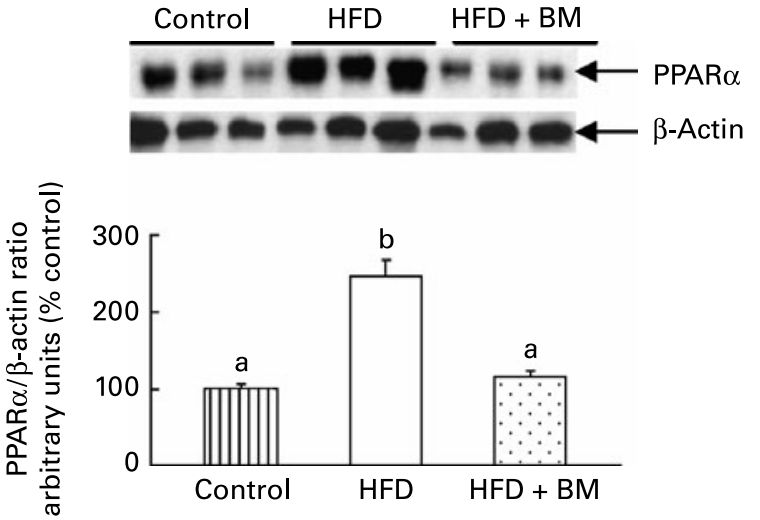

Fig. 6. Hepatic PPAR $\alpha$ protein levels in mice fed high-fat diet (HFD) and HFD + bitter melon juice (BMJ). The graph represents the densitometry scans of $52 \mathrm{kDa}$ band intensities. The $\beta$-actin band demonstrates the amount of protein loaded on the gel. Data are expressed as a percentage of control values, set at $100 \%$. Values are means with standard deviations depicted by vertical bars $(n 6){ }^{a, b}$ Mean values with unlike letters were significantly different $(P<0.05)$.

phosphorylated IRS proteins thereby blocking access to p85-p110 heterodimers. Thus a delicate balance between p85 monomers and p85-p110 heterodimers dictates PI3K activity $^{(26)}$. The present results indicate that BMJ significantly increased the amount of p110 associated with IRS-1 and IRS-2, but had no effect on p85 binding, suggesting activation of PI3K activity. BMJ had no effect on Akt tyrosine phosphorylation. However, we did not explore the phosphorylation of Akt at serine 473 and threonine 308 residues. BMJ-associated increased interactions between IRS and PI3K subunits could involve apoB degradation or reduction in the synthesis and secretion of apoB, since BMJ reduces plasma apoB in HFDfed mice as well as its secretion in HepG2 cells ${ }^{(15)}$. Future studies will investigate the role of BMJ-associated activation of AMP-activated protein kinase and lipoprotein secretion.

Besides apoB-100, BMJ also normalized plasma apoB-48 in HFD-fed mice. Intestinal over-production of apoB48-containing lipoproteins occurs in response to HFD leading to postprandial lipidaemia. Although, unlike man and hamsters, mice produce apoB-48-containing particles from both the liver and intestine, one cannot rule out the possibility that reduction of plasma apoB-48 observed in the present study may involve intestinal regulation of apoB-48 by BMJ.

HFD-fed mice in the present study demonstrate increase in plasma NEFA, which is attenuated by BMJ. The data support and extend the previous observations by Patsouris et al. ${ }^{(27)}$, which demonstrated that nuclear transcription factor PPAR $\alpha$ is significantly increased in HFD-fed mice possibly in response to an increased NEFA flux to the liver. Patsouris et al. ${ }^{(27)}$ further demonstrate that insulin suppresses PPAR $\alpha$ activation in hepatocytes and that hepatic up-regulation of PPAR $\alpha$ by HFD may be associated with attenuation of insulin signalling-associated increases in plasma NEFA. Recent studies by Ameen et al. ${ }^{(28)}$ demonstrated that in mice, Wy 14,643, a PPAR $\alpha$ agonist, increased apoB-100 secretion despite decreased TAG synthesis, specifically through activation of microsomal TAG transfer protein mRNA transcription and protein expression. We have previously demonstrated that reduction in apoB secretion was in part associated with reductions in TAG synthesis as well as microsomal TAG transfer protein mRNA gene expression in HepG2 cells treated with BMJ. In the current study, although we did not analyse hepatic microsomal TAG transfer protein expression, reduction in PPAR $\alpha$ expression paralleled the reduction in hepatic and plasma TAG as well as plasma apoB-100 secretion in mice fed $\mathrm{BMJ}$ as compared to those receiving HFD alone. The present results are, however, contradictory to those of Chao \& Huang ${ }^{(29)}$ who demonstrated that $72 \mathrm{~h}$ treatment of H4IIEC3 rat liver cells with BMJ extract (100 and $150 \mathrm{mg} / \mathrm{l}$ ) activated transcriptional factors such as PPAR $\alpha$ and PPAR $\gamma$, possibly contributing to its hypolipidaemic and hypoglycaemic effects. Nevertheless, BMJ-associated reductions in PPAR $\alpha$ protein levels were not below those of untreated control animals. Alternatively, the differences in PPAR $\alpha$ could arise due to the differences in the models employed and the fact that BMJ was tested in conjunction with HFD as well as the differences in the in vitro and in vivo metabolism of $\mathrm{BMJ}$.

Although HFD significantly increased liver weight and hepatic TAG levels, BMJ had no effect on liver weight, but significantly lowered hepatic TAG mass in HFD-fed mice. Similar BMJ-associated reduction in hepatic TAG mass without a change in liver weight has been observed in rats fed BM pulp ${ }^{(30)}$ or $\mathrm{BMJ}^{(4,22,31)}$ and has been attributed to increased clearance of hepatic TAG by BM through increased $\beta$-oxidation of NEFA.

Traditionally, diabetic subjects are recommended to consume about 30-60 g fresh BMJ twice a day. However, there are no clinical studies that demonstrate an effective physiological or pharmacological dosage of BM consumption by man. Nevertheless, BMJ preparations from independent laboratories have demonstrated comparable beneficial effects not only on glucose metabolism, but also on plasma and hepatic lipids ${ }^{(5,30)}$. Earlier studies by Chen et al. ${ }^{(7)}$ and Chen \& $\mathrm{Li}^{(22)}$ did not observe any dose-dependent effects of BMJ on body weight and metabolic parameters of glucose and lipid metabolism. With HFD, the maximum effects on metabolic parameters were observed at a dose of $1.5 \%$ of lyophilized BMJ (w/w) fed ad libitum ${ }^{(7)}$. The BM dose of $1.5 \%$ lyophilized juice was based on published literature mentioned earlier ${ }^{(7)}$. In the present study, $1.5 \%$ BMJ had no detrimental effects on liver function as measured by serum aspartate aminotransaminase, alanine amino transferase and lactate dehydrogenase levels as reported in previous studies ${ }^{(32)}$.

Although BM contains numerous chemicals, the active hypoglycaemic and hypolipidaemic compounds are unknown. Compounds isolated from the fruit and seeds of the BM plant that are believed to contribute to its hypoglycaemic activity include charantin (a steroid glycoside) and polypeptide 'p' or plant insulin (a 166 residue insulin mimetic peptide) ${ }^{(33)}$. $\mathrm{BM}$ is also known to contain additional glycosides such as mormordin, vitamin $\mathrm{C}$, carotenoids, flavanoids and polyphenols ${ }^{(34,35)}$. Recent studies indicate that cucurbutanoid compounds are the active principals of BM which possess hypoglycaemic properties ${ }^{(36)}$.

So far, only a few, non-randomized clinical studies have investigated the anti-diabetic effects of $\mathrm{BM}$ in $\operatorname{man}^{(37-41)}$. It is therefore crucial to conduct adequately powered, randomized, placebo-controlled clinical trials before BM can be recommended as an effective alternative and/or complementary 
therapy for diabetic dyslipidaemia. Administration of BM with other hypoglycaemic/hypolipidaemic agents must be performed under medical supervision and monitoring, due to its hypoglycaemic properties ${ }^{(37,42)}$. Some negative side-effects such as diarrhoea and hepatotoxicity in man have been noted $^{(40)}$, which could be due to excessive consumption.

The present data are the first to demonstrate that the primary mechanism of BMJ action to restore hepatic glucose and lipid metabolism is associated with increased post-IR signal transduction linked to tyrosine phosphorylation of IR $\beta$ and IRS proteins thereby leading to PI3K activation. Further studies are warranted not only to characterize and identify the active ingredients of BM that regulate lipid metabolism, but also to standardize physiologically relevant dosage in man.

\section{Acknowledgements}

This work was supported partially by US Public Health Service grants from the National Institutes of Health (NIH), Research Centers in Minority Institutions (RCMI, G12 RR003061), National Center for Research Resources (NCRR, P20 RR011091), National Center for Complementary and Alternative Medicine (NCCAM, R21AT003719) and the Hawaii Community Foundation (20041652). We thank Dr Jimmy Efird of the UHM-RCMI Biostatistical Core for statistical analysis and Ms Jodi Cope for technical assistance with animal care. All the authors have approved the paper and have no conflicts of interest. P. V. N. developed ideas, designed experiments, secured funding and wrote the manuscript. Y. K. L. and M. M. performed 90 and $10 \%$ of the experiments, respectively. K. A. assisted in standardizing techniques and reviewing the manuscript. V. R. N. provided critical insights in the development of this project and the process of revising draft versions of the manuscript.

\section{References}

1. Abascal K \& Yarnell E (2005) Using bitter melon to treat diabetes. Altern Complement Med 1, 179-184.

2. Yadav UC, Moorthy K \& Baquer NZ (2005) Combined treatment of sodium orthovanadate and Momordica charantia fruit extract prevents alterations in lipid profile and lipogenic enzymes in alloxan diabetic rats. Mol Cell Biochem 268, 111-120.

3. Chan LL, Chen Q, Go AG, Lam EK \& Li ET (2005) Reduced adiposity in bitter melon (Momordica charantia)-fed rats is associated with increased lipid oxidative enzyme activities and uncoupling protein expression. J Nutr 135, 2517-2523.

4. Senanayake GV, Maruyama M, Shibuya K, Sakono M, Fukuda N, Morishita T, Yukizaki C, Kawano M \& Ohta H (2004) The effects of bitter melon (Momordica charantia) on serum and liver triglyceride levels in rats. J Ethnopharmacol 91, 257-262.

5. Senanayake GV, Maruyama M, Sakono M, Fukuda N, Morishita T, Yukizaki C, Kawano M \& Ohta H (2004) The effects of bitter melon (Momordica charantia) extracts on serum and liver lipid parameters in hamsters fed cholesterol-free and cholesterol-enriched diets. J Nutr Sci Vitaminol (Tokyo) 50, 253-257.

6. Chaturvedi P, George S, Milinganyo M \& Tripathi YB (2004) Effect of Momordica charantia on lipid profile and oral glucose tolerance in diabetic rats. Phytother Res 18, 954-956.

7. Chen Q, Chan LL \& Li ET (2003) Bitter melon (Momordica charantia) reduces adiposity, lowers serum insulin and nor- malizes glucose tolerance in rats fed a high fat diet. $J$ Nutr 133, 1088-1093.

8. Virdi J, Sivakami S, Shahani S, Suthar AC, Banavalikar MM \& Biyani MK (2003) Antihyperglycemic effects of three extracts from Momordica charantia. J Ethnopharmacol 88, 107-111.

9. Bonn V, Cheung RC, Chen B, Taghibiglou C, Van Iderstine SC \& Adeli K (2002) Simvastatin, an HMG-CoA reductase inhibitor, induces the synthesis and secretion of apolipoprotein AI in HepG2 cells and primary hamster hepatocytes. Atherosclerosis 163, 59-68.

10. Carpentier A, Taghibiglou C, Leung N, Szeto L, Van Iderstine SC, Uffelman KD, Buckingham R, Adeli K \& Lewis GF (2002) Ameliorated hepatic insulin resistance is associated with normalization of microsomal triglyceride transfer protein expression and reduction in very low density lipoprotein assembly and secretion in the fructose-fed hamster. J Biol Chem 277, 28795-28802.

11. Sparks JD, Phung TL, Bolognino M \& Sparks CE (1996) Insulin-mediated inhibition of apolipoprotein $\mathrm{B}$ secretion requires an intracellular trafficking event and phosphatidylinositol 3-kinase activation: studies with brefeldin A and wortmannin in primary cultures of rat hepatocytes. Biochem J 313, 567-574.

12. Avramoglu RK \& Adeli K (2004) Hepatic regulation of apolipoprotein B. Rev Endocr Metab Disord 5, 293-301.

13. Chong T, Naples M, Federico L, Taylor D, Smith GJ, Cheung RC \& Adeli K (2006) Effect of rosuvastatin on hepatic production of apolipoprotein B-containing lipoproteins in an animal model of insulin resistance and metabolic dyslipidemia. Atherosclerosis 185, 21-31.

14. Taghibiglou C, Rashid-Kolvear F, Van Iderstine SC, Le-Tien H, Fantus IG, Lewis GF \& Adeli K (2002) Hepatic very low density lipoprotein-ApoB overproduction is associated with attenuated hepatic insulin signaling and over expression of protein-tyrosine phosphatase 1B in a fructose-fed hamster model of insulin resistance. J Biol Chem 277, 793-803.

15. Nerurkar PV, Pearson L, Efird JT, Adeli K, Theriault AG \& Nerurkar VR (2005) Microsomal triglyceride transfer protein gene expression and ApoB secretion are inhibited by bitter melon in HepG2 cells. J Nutr 135, 702-706.

16. Raj SK, Khan MS, Singh R, Kumari N \& Prakash D (2005) Occurrence of yellow mosaic geminiviral disease on bitter gourd (Momordica charantia) and its impact on phytochemical contents. Int J Food Sci Nutr 56, 185-192.

17. Saha PK, Kojima H, Martinez-Botas J, Sunehag AL \& Chan L (2004) Metabolic adaptations in the absence of perilipin: increased beta-oxidation and decreased hepatic glucose production associated with peripheral insulin resistance but normal glucose tolerance in perilipin-null mice. $J$ Biol Chem 279, 35150-35158.

18. Scribner K, Gadbois T, Gowri M, Azhar S \& Reaven G (2000) Masoprocol decreases serum triglyceride concentrations in rats with fructose-induced hypertriglyceridemia. Metabolism 49, $1106-1110$.

19. Hui DY (2003) HIV protease inhibitors and atherosclerosis. $J$ Clin Invest 111, 317-318.

20. Messier C, Whately K, Liang J, Du L \& Puissant D (2007) The effects of a high-fat, high-fructose, and combination diet on learning, weight, and glucose regulation in C57BL/6 mice. Behav Brain Res 178, 139-145.

21. Avramoglu RK, Basciano H \& Adeli K (2006) Lipid and lipoprotein dysregulation in insulin resistant states. Clin Chim Acta 368, $1-19$.

22. Chen Q \& Li ET (2005) Reduced adiposity in bitter melon (Momordica charantia) fed rats is associated with lower tissue triglyceride and higher plasma catecholamines. Br J Nutr 93, 747-754.

23. Nerurkar PV, Lee YK, Linden EH, Lim S, Pearson L, Frank J \& Nerurkar VR (2006) Lipid lowering effects of Momordica 
charantia (Bitter Melon) in HIV-1-protease inhibitor-treated human hepatoma cells, HepG2. Br J Pharmacol 148, 1156-1164.

24. Shepherd PR, Withers DJ \& Siddle K (1998) Phosphoinositide 3-kinase: the key switch mechanism in insulin signaling. Biochem J 333, 471-490.

25. Arimura N, Horiba T, Imagawa M, Shimizu M \& Sato R (2004) The peroxisome proliferator-activated receptor gamma regulates expression of the perilipin gene in adipocytes. J Biol Chem 279, 10070-10076.

26. Ueki K, Fruman DA, Yballe CM, Fasshauer M, Klein J, Asano T, Cantley LC \& Kahn CR (2003) Positive and negative roles of p85 alpha and p85 beta regulatory subunits of phosphoinositide 3-kinase in insulin signaling. J Biol Chem 278, 48453-48466.

27. Patsouris D, Reddy JK, Muller M \& Kersten S (2006) Peroxisome proliferator-activated receptor alpha mediates the effects of high-fat diet on hepatic gene expression. Endocrinology 147, 1508-1516.

28. Ameen C, Edvardsson U, Ljungberg A, Asp L, Akerblad P, Tuneld A, Olofsson SO, Linden D \& Oscarsson J (2005) Activation of peroxisome proliferator-activated receptor alpha increases the expression and activity of microsomal triglyceride transfer protein in the liver. J Biol Chem 280, 1224-1229.

29. Chao CY \& Huang CJ (2003) Bitter gourd (Momordica charantia) extract activates peroxisome proliferator-activated receptors and up regulates the expression of the acyl CoA oxidase gene in H4IIEC3 hepatoma cells. J Biomed Sci 10, 782-791.

30. Jayasooriya AP, Sakono M, Yukizaki C, Kawano M, Yamamoto K \& Fukuda N (2000) Effects of Momordica charantia powder on serum glucose levels and various lipid parameters in rats fed with cholesterol-free and cholesterol-enriched diets. J Ethnopharmacol 72, 331-336.

31. Ahmed I, Lakhani MS, Gillett M, John A \& Raza H (2001) Hypotriglyceridemic and hypocholesterolemic effects of antidiabetic Momordica charantia (karela) fruit extract in streptozotocin-induced diabetic rats. Diabetes Res Clin Pract 51, $155-161$.

32. Abd El Sattar El Batran S, El-Gengaihi SE \& El Shabrawy OA (2006) Some toxicological studies of Momordica charantia L. on albino rats in normal and alloxan diabetic rats. $J$ Ethnopharmacol 108, 236-242.

33. Khanna P, Jain SC, Panagariya A \& Dixit VP (1981) Hypoglycemic activity of polypeptide-p from a plant source. J Nat Prod 44, 648-655.

34. Jantan I, Rafi IA \& Jalil J (2005) Platelet-activating factor (PAF) receptor-binding antagonist activity of Malaysian medicinal plants. Phytomedicine 12, 88-92.

35. Anila L \& Vijayalakshmi NR (2000) Beneficial effects of flavonoids from Sesamum indicum, Emblica officinalis and Momordica charantia. Phytother Res 14, 592-595.

36. Harinantenaina L, Tanaka M, Takaoka S, Oda M, Mogami O, Uchida M \& Asakawa Y (2006) Momordica charantia constituents and antidiabetic screening of the isolated major compounds. Chem Pharm Bull (Tokyo) 54, 1017-1021.

37. Welihinda J, Karunanayake EH, Sheriff MH \& Jayasinghe KS (1986) Effect of Momordica charantia on the glucose tolerance in maturity onset diabetes. J Ethnopharmacol 17, 277-282.

38. Leatherdale BA, Panesar RK, Singh G, Atkins TW, Bailey CJ \& Bignell AH (1981) Improvement in glucose tolerance due to Momordica charantia (karela). Br Med J (Clin Res Ed) 282, $1823-1824$

39. Srivastava Y, Venkatakrishna-Bhatt H, Verma Y, et al. (1993) Antidiabetic and adaptogenic properties of Momordica charantia extract. An experimental and clinical evaluation. Phytother Res 7, 285-289.

40. Basch E, Gabardi S \& Ulbricht C (2003) Bitter melon (Momordica charantia): a review of efficacy and safety. Am J Health Syst Pharm 60, 356-359.

41. Tuekpe MK, Todoriki H, Sasaki S, Zheng KC \& Ariizumi M (2006) Potassium excretion in healthy Japanese women was increased by a dietary intervention utilizing homeparcel delivery of Okinawan vegetables. Hypertens Res 29, 389-396.

42. Ahmed N, Hassan MR, Halder H \& Bennoor KS (1999) Effect of Momordica charantia (Karolla) extracts on fasting and postprandial serum glucose levels in NIDDM patients. Bangladesh Med Res Counc Bull 25, 11-13. 